\begin{tabular}{|c|c|c|}
\hline & $\begin{array}{l}\text { International Journal of Environment, Agriculture and Biotechnology } \\
\qquad \text { Vol-6, Issue-3; May-Jun, } 2021\end{array}$ & $\theta=$ \\
\hline IJEAB & $\begin{array}{c}\text { Journal Home Page Available: } \underline{\text { https://ijeab.com/ }} \\
\text { Journal DOI: } \underline{10.22161 / \text { ijeab }}\end{array}$ & \\
\hline
\end{tabular}

Article

Peer-Reviewed Journal

\title{
Cancer Therapy with CRISPR/Cas9: Prospects and Challenges
}

\author{
Muhammad Fahad Chaudhary ${ }^{1 *}$, Unsa Saleem ${ }^{1}$, Samia Khan ${ }^{1}$, Rabiya Khan ${ }^{2}$, \\ Muhammad Asim ${ }^{1}$, Fakhra Azam ${ }^{1}$, Mariam Sharif ${ }^{3}$, Zainab $^{1}$, Ali Abbas Dilawar ${ }^{1}$, \\ Muhammad Jehanzaib ${ }^{1}$
}

\author{
${ }^{1}$ Department of Microbiology, Government College University Faisalabad, Pakistan \\ ${ }^{2}$ Department of Medical Lab Technology, Government College University Faisalabad, Pakistan \\ ${ }^{3}$ Institute of Microbiology, University of Agriculture Faisalabad, Pakistan \\ *Corresponding Author: Fahadchaudhary1999@gmail.com
}

Received: 04 Apr 2021; Received in revised form: 21 May 2021; Accepted: 01 Jun 2021; Available online: 17 Jun 2021

(C2021 The Author(s). Published by Infogain Publication. This is an open access article under the CC BY license

(https://creativecommons.org/licenses/by/4.0/).

\begin{abstract}
Globally, Cancer is believed to be second biggest reason of mortality and one of the significant social as well as economic liabilities. Despite our advance at molecular level in comprehension of cancer, more therapeutic tools and tactics are needed to exploit this advance. The CRISPER/Cas 9 genome modifying approach has lately appeared as an effective cancer therapy method due to its high accuracy and efficiency. CRISPER/Cas9 has enormous clinical potential in discovering new targets for cancer treatment and also to dismember genetic-chemical interaction thus helping us to understand the response of tumor to the treatment by drugs. Additionally, Cas9/CRISPER can also be used in cancer immunotherapeutic applications by engineering immune cells and oncolytic viruses. Perhaps the most important therapeutic application of Cas9/CRISPER is its ability to edit genes with great precision both in animal models and humans. In this review, we will debate and explore some important concerns of using CRISPER/Cas 9 in remedial settings and some vital hurdles that are needed to overcome before it is used for a clinical trial for a polygenic and complex ailment like cancer.
\end{abstract}

Keywords-Advance therapy; CRISPER; Cas9; Genome engineering; Cancer models.

\section{BENEFITS AND TECHNIQUES OF CRISPER GENOME MODIFICATION}

The discovery of clustered regularly interspaced short palindromic repeats (CRISPR) (Ishino, Shinagawa, Makino, Amemura, \& Nakatura, 1987; Van Soolingen, De Haas, Hermans, Groenen, \& Van Embden, 1993)and their role as an adaptive prokaryotic immune system in combination with CRISPR-associated (Cas) genes (Bolotin, Quinquis, Sorokin, \& Dusko Ehrlich, 2005; van der Oost, Jore, Westra, Lundgren, \& Brouns, 2009)helped open the way to be used as a potent tool for genome related engineering (Jinek et al., 2012; Mali, Esvelt, \& Church, 2013). The Cas9/CRISPR is considered to be the major bio-tech breakthrough of the century for its precision, efficiency, and ease, and has paved the way for more accurate genome modification and in vivo visualization. Generally, Cas9/CRISPR has appeared to have unparalleled therapeutic possibility for studying and targeting disorder, as well as opening up new drug development pathways. More precisely, it heralds the arrival of novel diagnostic and therapeutic approaches.

Gene editing techniques operate by generating doublestrand breaks (DSBs) in particular genome sections, which are then repaired by cellular processes. RNA-guided DNA targeting CRISPR/Cas, in comparison to earlier genome editing methods such as zinc-finger nucleases (ZFNs) and transcription activator-effector nucleases (TALENs), was instantly and extensively embraced by clinicians due to its 
cost-effectiveness, adaptibility, and convenient for use, thus innovating the work of genome related engineering. Cas9 technology is based on a bacterial and archaeal immune defence mechanism that protects the host from viruses and phages that target nucleic acids(Barrangou et al., 2007). According to their most popular classification, CRISPER/Cas system is classified into three main types, all with various sub classification(Makarova et al., 2011). The most widely used gene editing system is the type II CRISPR/Cas system, which consists of three components: an endonuclease (Cas9), a CRISPR RNA (crRNA), and a transactivatingcrRNA (tracrRNA) (Jinek et al., 2012). The guide RNA (gRNA) is a duplex structure formed by the crRNA and tracrRNA molecules which can be substituted using a fused synthetic chimeric single gRNA (sgRNA), making CRISPR/Cas9 easier to use in genome engineering(Jinek et al., 2012).The sgRNA carries a special sequence of approximately 20 base-pairs (bp) and is intended to complement the DNA site that is targeted, and it should be accompanied by a small length DNA sequence known as the "protospacer-adjacent motif" (PAM), that is required for affinity of Cas9 protien. The expression of sgRNA as well as Cas9 nuclease in the cell create a ribonucleoprotein (RNP) complex, which is directed to a target DNA site by the sgRNA. Cas9 specifically cleaves the DNA to generate a DSB after the sgRNA binds to the target sequence using Watson-Crick base-pairing. The cleavage takes place inside the protospacer, three nucleotides upstream of the PAM, resulting in blunt ends. Cas9 active-site motifs RuvC and $\mathrm{HNH}$, which acts on the (-) and (+) strands respectively, are liable for splitting of opposite DNA strands (Gasiunas, Barrangou, Horvath, \& Siksnys, 2012; Mojica, DíezVillaseñor, García-Martínez, \& Almendros, 2009). The machinery of cell mends the DSB using one out of two major mechanisms, depending on the cell state and the availability of a repair template: homology-directed repair (HDR) or non-homologous end joining (NHEJ)(Differ, Cobb, Fishman-lobell, \& Habert, 1992; Liang, Han, Romanienko, \& Jasin, 1998). The HDR pathway works at the DSB site by recombining a template donor DNA, ensuing repairment that must be accurate. Relevant sequences or mutations may be introduced into a target area of the genome using homology-directed repair. A more common NHEJ pathway is error-prone mechanism that generates frameshift mutations at the DSB siteby randomly inserting or deleting nucleotides (indels). It may thus be used to cause specific gene knockouts (Fig. 1).

Cas9/CRISPER techniques permit for accurate as well as effective splitting of desired targeted DNA sequences, and it has greatly enabled genome editing due to the relative ease along with clarity of constructing
sgRNAs. The use of diverse sgRNAs allows this technology to be multiplexed, which is an additional benefit. Only the CRISPR/Cas9 method, among genome editing nucleases, can edit several loci at the same time by adding sgRNAs to different locations(Jakočinas et al., 2015; Li, Teng, Li, \& Zhou, 2013). Where two sgRNAs are used in the same cell, minor deletions(Wyman et al., 2013), complex rearrangements(P. S. Choi \& Meyerson, 2014; Torres, Martin, et al., 2014), and even wholechromosome suppression can occur(Adikusuma, Williams, Grutzner, Hughes, \& Thomas, 2017). One more significant benefit of Cas9/CRISPR is its adaptability: amendments and personalization of Cas9/CRISPR modules and also interactors have upgraded the system's precision and efficacy while also broadening its scope of applications beyond editing (Dominguez, Lim, \& Qi, 2016).

CRISPR technology's DNA precision has become a main focus in the work field and the existence of offtarget activity is shown by many experiments(Tsai \& Joung, 2016). As a result, a number of tactics have been devised to reduce the products that are off-target. Out of various methods, one method uses a cellular delivery of in vitro-assembled RNP complexes instead of plasmid delivery, which generates longer-lived Cas9 and sgRNA expression, as well as a higher ratio of on-target:off-target editing of genes in the cells of mammals and vastly effective editing(Kim, Kim, Cho, Kim, \& Kim, 2014; Torres-Ruiz et al., 2017). Other approaches include using Cas9 variants that are light or small molecule inducible(Davis, Pattanayak, Thompson, Zuris, \& Liu, 2015; Nihongaki, Kawano, Nakajima, \& Sato, 2015), broken Cas9 variants (for safe rebuilding), and Cas9 which are regulated allosterically(Oakes et al., 2016). The Cas9 was modified to induce break just one strands of DNA, researchers were able to use pair of Cas9 nickases directed by two different gRNAs aiming at the same locus but on opposing DNA strands. This technique creates highly precise DNA splitting with efficacy similar to traditional Cas9/CRISPR but with less far-off incidents(Ran et al., 2013). A similar strategy uses two Cas9 which are catalytically inactivated mutants fused to (fCas9) FokI nuclease (directed by two opposing gRNAs projecting at the same position), in which the only dimer (fCas9) is functional. In human cells, Fokl nucleases were found to alter a specified location with $>150$-folds greater accuracy than Cas9 which are wild-type nucleases(Guilinger, Thompson, \& Liu, 2014). Finally, study of Cas9 mutations to improve specificity revealed that 3-4 modified point variations can neutralize electrostatic interaction between its targeted DNA and Cas9, resulting in a considerable improvement in the specificity of its action(Kleinstiver et al., 2016; Slaymaker et al., 2016). Although CRISPR/Cas9 
technology has a number of benefits over prior genome editing programmable nucleases, but it also has some disadvantages. CRISPR/Cas9 performance and sequence specificity requires to be developed beyond. Effects that are off-target must also be minimized, and developing a CRISPR/Cas9 delivery system that is reliable, stable, and cell-specific remains a major challenge.

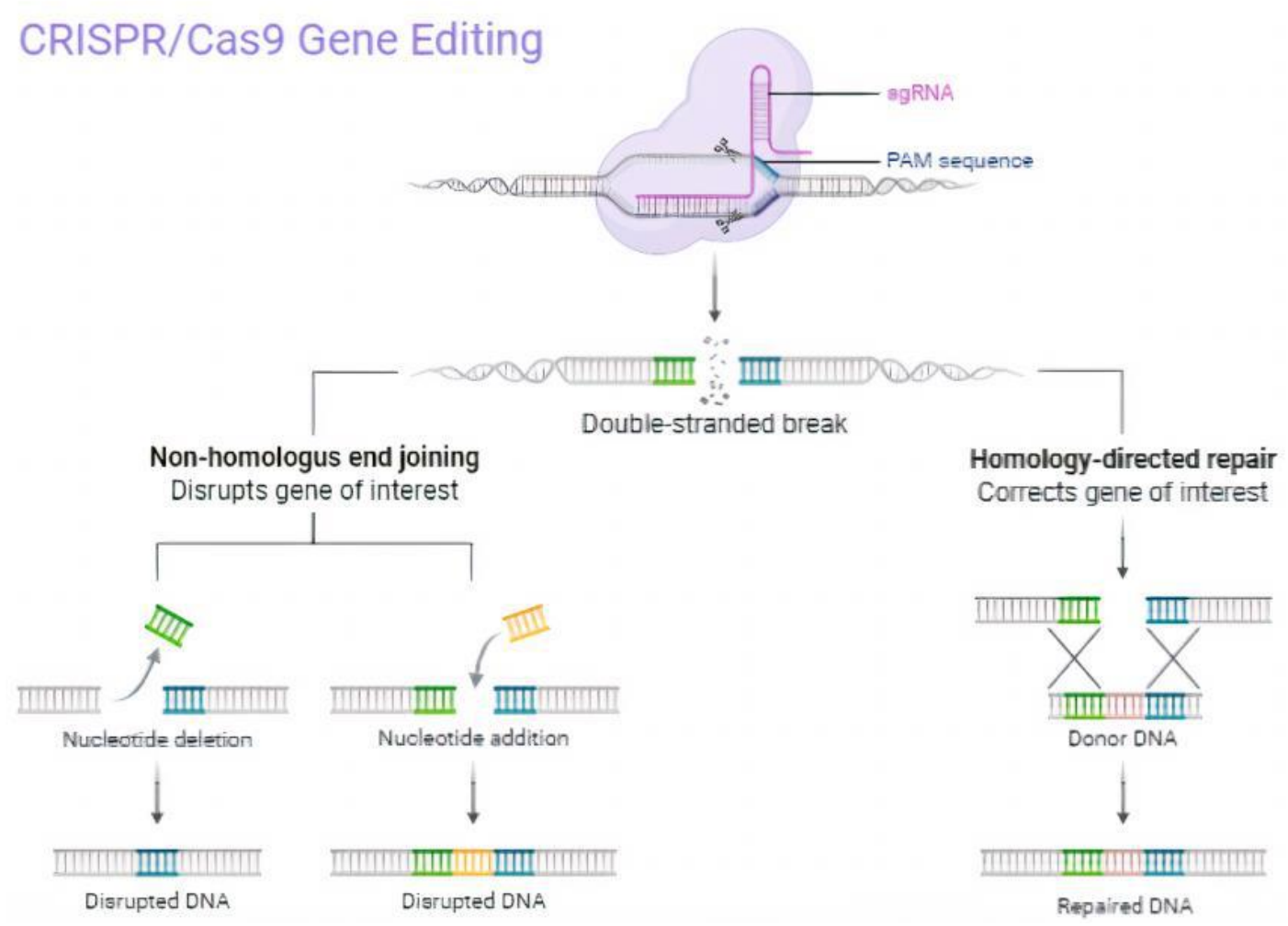

Fig. 1: Mechanism of action of CRISPER/Cas9. When the protein Cas9 attaches to sgRNA, it forms a (RNP) ribonucleoprotein complex. After recognizing the protospacer-adjacent motif(PAM) sequence adjoining to the selected sequence, this complex fuse to the genomic target sequence with base-pairing complementarity and accurately splits doublestranded DNA. When breaks occur in double-strand, the NHEJ or HDR channels are triggered. In the absence of homologous repair template, NHEJ usually results in deletions or insertions of arbitrary base pairs disturbing the selected sequence. By supplying a donor DNA template and using the homology-driven repair pathway, specific genome edition can also be achieved.

\section{DRUG EXPLORATION WITH CRISPER}

Drug discovery and production is a lengthy and complicated procedure that involves recognizing novel products and presenting them in the market. Typically, the procedure starts with the proposition that disrupting certain biological target will result in one useful outcome that will alter disease progression. These targets should be confirmed in physiologically pertinent models of animals prior to clinical models whose pharmacological adjustment may lead to the required therapeutic impact.

In cancer studies, drug formulation aims to recognize molecules against genetic defects in tumor suppressor gene as well as oncogenes that lead to tumor formation. Various prominent examples contains vemurafenib, that directs at BRAF V600E mutant variations in melanoma; Osimertinib, for EGFR-mutated non-small cell lung cancer treatment; or imatinib, that targets fusion of ABL1-BCR in long-term myeloid leukemia(Fleuren, Zhang, Wu, \& Daly, 2016).

Drug discovery program can be aided with genome engineering techniques by identifying genes that are accountable for specific ailment. The process of identifying and confirming high-value targets is laborious and time-consuming, but the CRISPER/Cas9 method has the ability to speed it up.The quick and efficacious generation of accurate disease models, both animal as well as cellular, using Cas9/CRISPER modification has an approving effect on discovery of drug because it is an instant way for active drug testing by identifying selected molecules that are activated or inhibited to induce or prevent disease(Ahmad \& Amiji, 2018). 


\section{DRUG TARGET EXPLORATION USING CRISPER/CAS9 LIBRARY SCREENS}

The discovery of genes that are not known and the finding out about their role are normally done using highthroughput genetic screening platforms. Screening of mutations has been utilized to recognize fundamental biotic procedures and pathways for signaling, and it can also be used to establish genes which are accountable for a specified phenotype. The major restraint of mutagenesis screening for targeted discovery of drug with the mutations that are not known is the propagation of heterozygous mutants. Targeted RNA interference (RNAi) is a way to overcome this constraint. High-production RNAi genomic library tests may provide vital details on connections among single genes and functional loss phenotypes, however there are yet some drawbacks, such as useless knockdown and significant far off targets(Ahmad \& Amiji, 2018). CRISPER/Cas9 has some leads over RNAi including complete inactivation, whole genome targeting capability including introns, promoter, enhancer and intergenic regions, and high reproducibility(Wang, Wei, Sabatini, \& Lander, 2014).The expertise acquired through the creation of operative RNAi approaches has aided the rampant growth of CRISPER/Cas9 libraries in recent years. In 2013, these CRISPER libraries in the beginning were said to be more effective than RNAi libraries (Wang et al., 2014).

Three distinct kind of genome-wide libraries are lately in use: (1) To find out new biological mechanism encompassing cell survival signals and drug resistance, CRISPER-based loss-of-function (CRISPER knock-out)is used (Ribeiro, 2014); (2) CRISPER based new gene activation (CRISPERa) is vital in testing for gain of function (Joung et al., 2017); and (3) Screening for loss of function can be accomplished by using CRISPER based gene inhibition (CRISPERi) (Luo, 2016). CRISPERa and CRISPERi libraries, unlike CRISPER knock-out libraries, use catalytically ineffective Cas9 in conjunction with regulative cofactors like VP64 (activation)(Maeder et al., 2013)or Kruppel associated box (KRAB) repressin (inhibition) (Gilbert et al., 2014)or other factors like SAM or SunTag(Chavez et al., 2016; Konermann et al., 2015), developed to speed up CRISPERa activity. (Fig. 2).

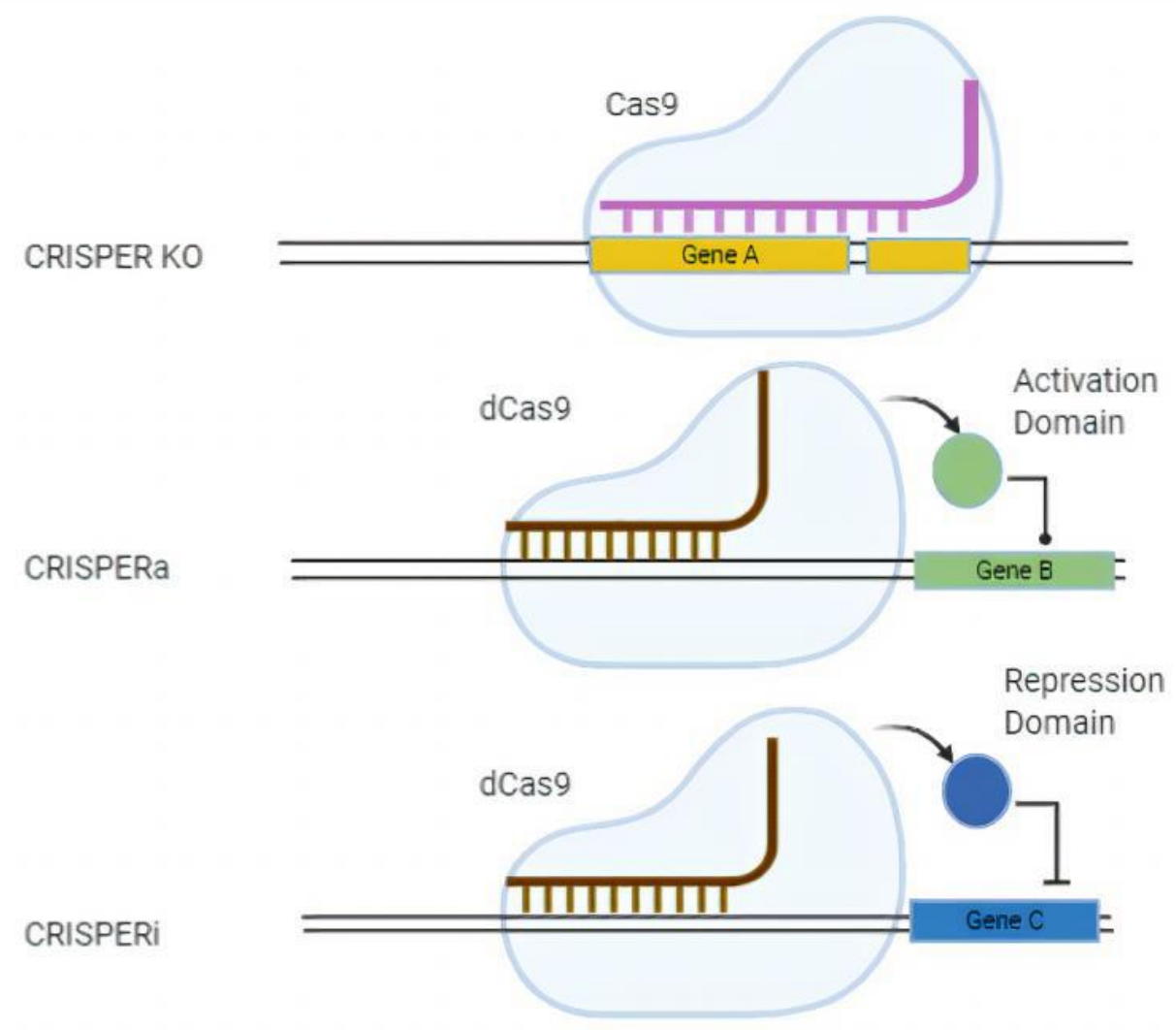

Fig. 2: Three main techniques for transcriptional modulation: (1) CRISPER Knock-out, for production of protiens that are non-functional or knock-out a specific gene by using wild type CRISPER system; (2) CRISPER activation, It uses a catalytically deactivated variant of Cas 9 enzyme in conjunction with various activators domains to produce specific gene activation(SunTag, SAM, VPR); (3) CRISPER repression or deactivation, by fusing repressor domain (KRAB) with catalytically inactivated dCas 9 . 


\section{DRUG RESISTANCE AND CRISPER/CAS9}

Finding of genes that play a part in resistance of drugs is a crucial implementation of CRISPER/Cas9 in drug finding. Conventionally, worldwide mutagenesis across a cell population is used to evaluate the mechanism of resistance of anticancer agents. Only cells carrying the mutations that spoil the action of drug would survive in ensuing usage of drug to be tested. However, generation of significant number of false positives is the drawback of this approach (Guichard, 2017).

CRISPER/Cas9 screens are the most appropriate tactic for the detection of gene deletion that is linked with drug resistance. Cells resistant to the desired drug are revealed to a pool of CRISPER/Cas9 gRNAs that targets genome in a way that each cell has one guide that knocks out single gene. Subsequent examination of cells that becomes vulnerable by exposure of drugs, point out the genes that confer drug resistance. Resistant genes can then be targeted with other drugs to evade exposure of resistance (Scott, 2018)E.g., HPRT1 gene interference through CRISPER/Cas9 editing generates 6-thioguannie resistant cells (Smurnyy et al., 2014).

\section{DRUG EFFECTIVENESS MODELS OF DISEASES}

In drug development, cells and animal models are of substantial importance. Before clinical testing on humans, experiments must be performed in models to test drug effectiveness and toxicity. Most subjects, encompassing cell as well as animal models, are not able to, however, accurately represent the condition observed in patients. Moreover, to generate subjects that precisely summarize the variety and complexity of disease are very expensive and time consuming process. Cancer cell line can be modified to accurately mimic the deviations seen in patients by using CRISPER/Cas9 and it is cheap as compared to standard protocol. Model of ovarian cancer of mice ID8 was altered to hinder TP53 and BRCA2 which ultimately resulted in increased sensitivity to inhibit PARP, is a good example (Walton et al., 2016).

The example above demonstrates clearly that CRISPER/Cas9 platform has become vital element of drug discovery in oncology. This mechanization has enhanced the finding and authentication of novel drug targets, as well as providing more accurate models of human diseases for evaluating safety of drug in a more prognostic way as well as reducing and combating drug resistance.

\section{CRISPER AS A CANCER-FIGHTING TOOL}

Although there is some advancement in past decade but significant number of people still die due to cancer which demonstrate the dire need for novel and more effective therapeutic options. CRISPER/Cas9 genome editing has a great potential in cancer therapeutic besides its use as a research tool. The regulation of endogenous gene expression is a probable application of CRISPER/Cas9 system in Cancer therapy. As discussed above, gRNAs can be used to recruit catalytically inactive dCas9 to specific target DNA sites(Friedland et al., 2013) and can also be utilized to activate or suppress particular target genes by fusing it with transcriptional activation or inhibition domain(Chen et al., 2013). Epigenome editing could be another therapeutic application based on linking dCas 9 to histone modifiers and proteins involved in altering DNA methylation (Klann et al., 2017). Finally, by specifically targeting tumor markers in cancer cells, it allows for the elimination of genetic changes that can contribute to tumor proliferation and/or metastatic capacity (Shachaf et al., 2004). However, the effectual delivery of CRISPER component in all the cancer cells is still a challenge.

Elaborate interaction between tumor, host and environment is needed for effective immunity against cancer cells because cancer is a complex disease. Immunotherapy, which targets PD-1 or increases immune action to cancer cells that has chimeric antigen therapy (CAR) therapy, has recently emerged as a promising treatment choice for cancer (Shachaf et al., 2004). Unlike chemotherapy or radiotherapy, Cancer immunotherapy has many benefits like durable activity, favorable benefits, and low risk ratio. The development of new generation therapy techniques is needed for cancers that are untreatable with traditional chemo or radiotherapy.

Viruses capable of oncolysis are becoming increasingly relevant in cancer related therapy.These can be modified genetically to attack only the cancer cells with deficient antiviral defense leaving normal cells intact. Virally motivated disruption of tumor cells has many mechanism including direct cellular lysis in which tumor antigen is released from dying cells which causes further immune stimulation (A. H. Choi, O'Leary, Fong, \& Chen, 2016). CRISPER/Cas9 mediated genome editing has enormous potential to be used in cancer therapeutics because it can be used to modify viruses capable of oncolysis for increased immune stimulation and optimized tumor selectivity. One example of genome modification for immunotherapy application is the generation of herpes simplex virus type 1 variants with improved lysis specifications by deleting of theICP34.5 neurovirulence and ICP6 (ribonucleotide reductase) genes(Goldsmith, 
Chen, Johnson, \& Hendricks, 1998). Furthermore, the ICP6 gene can be deleted to give selectivity which is replicative for cells with $\mathrm{P} 16^{\mathrm{INK} 41}$ tumour repressor gene deactivation, which is one of the major usual cancer insufficiencies(Aghi, Visted, DePinho, \& Chiocca, 2008). The wild type of adenovirus, in case of DNA tumor viruses, encodes protein (E1A) that can bind to $\mathrm{pRb}$, and therefore capturing the cycle of cell by release of transcription factor E2F. The release of this transcriptional factor E2F also causes an orderly activation of genes which are viral, resulting in the propagation of new viruses, which then cause the infected cell to lyse and release novel virus. Because cancerous cells usually contain genetic changes in the pathway named $\mathrm{Rb}$, the EIA gene has been knocked out of oncolytic adenoviruses to avoid replication.

Isolation and the in-vitro extension of tumor-specific $\mathrm{T}$ cells, accompanied by their reintroduction into the individual, are part of some immunotherapy methods such as Adoptive cell therapy (ACT). Many form of ACT are still under development which include engineered T-cells to efficiently recognize and attack tumor cells. This can be done by deleting PD-1 gene in T-cells( $\mathrm{Su}$ et al., 2016). Interaction of PD-1 and its ligand PD-L1 halts TLymphocytes propagation, effector function and their survival(Tseng et al., 2001), induce resistance of tumor cells to cytolytic T-lymphocyte reaction(Dong et al., 2002), as well as induce death of tumor specific Tcells(Iwai et al., 2002; Tsushima et al., 2007). The whole idea of this approach is that when PD-1 gene is deleted from T-cells in-vitro by using CRISPER/Cas9 and then subsequently reintroduced into the patient, those genedeleted T-cells will home tumor and turn on immune system which may eliminate tumor. One powerful tool for anti-tumor treatment is immune checkpoint blockade which include deletion of gene on anti-PD-1/PD-L1 and anti-CTLA-4 antibodies. This method has led the way to the innovation in the treating of many kinds of modern tumor by avoiding exhaustion triggered through checkpoint molecules. With PD-1 knockout T-cells, this technique is being investigated in six clinical studies for prostate, stomach, bladder, lymphoma, renal cell carcinoma, and hepatocellular carcinoma(Fellmann, Gowen, Lin, Doudna, \& Corn, 2017).

Production of the next generation CAR T-cells that are genetically altered to present tumor-targeting receptor is another impressive anti-cancer immune therapy that has a great potential for the treatment of hematological and solid cancers (Maus, Grupp, Porter, \& June, 2014). Intracellular chimeric signaling domain that is capable of activating $\mathrm{T}$ cells and an extracellular binding domain that recognize a highly specific antigen for and strongly expressed on tumor cells, together constitute a chimeric antigen receptor (CAR) and both domains work in combination to reprogram T-cell facilitated killing of Tumor cells. In 2016, an oncologist team led by LU You at Sichuan University in China became the first to inject T-cells modified by CRISPER/Cas9 to disable PD-1 into patients with aggressive lung cancer(Cyranoski, 2016). Although ACT therapies are of great potential in the treatment of leukemia and lymphoma, but some individuals died while conducting trial phases due to neurotoxicity and cytokine release syndrome (Gauthier \& Turtle, 2018). At this moment, FDA has approved CAR T-cell therapy only for the treatment of relapsed and refractory B-cell acute lymphoblastic leukemia in paediatric and young adults (Kansagra \& Litzow, 2017).

\section{GENE MODIFICATION BIOLOGICAL TECHNIQUES FOR IN-VIVO DELIVERY}

Development of effective and safe method for delivering gene editing element to the tumor cells as well as metastatic sites is still a defiance for the upcoming application of gene modification techniques such as systems like CRISPER/Cas9. Hitherto, In-Vitro gene editing has been performed mainly in hematopoietic precursors or T-cells. Developing efficient and safe methods for In-Vivo delivery in somatic cells is essential to widen the scope of CRISPER-based therapy. These delivery hurdles can be overcome by developing novel viral and non-viral systems (Kay, 2011; Torres, Garcia, Jimenez, Rodriguez, \& Ramirez, 2014).

Adeno-associated virus (AAV), lentivirus and adenovirus can be used as a viral delivery system for CRISPER/Cas9 components (Yin, Kauffman, \& Anderson, 2017).Presently, the latest approach for in-vivo delivery of gene make use of Adeno-associated viruses (AAVs)(Yin et al., 2016). There are many reasons for which AAVs are considered to be an outstanding vehicle for gene therapy: (1) AAV incite little or no immune response; (2) extensive variety of serotypes for infection of different cell types are known; (3) these viruses do not cause any disease in humans (Daya \& Berns, 2008). Furthermore, these viruses have been tested for their efficiency and safety in clinical trials (Kotterman \& Schaffer, 2014); and they have been favorably put to use in models of mice(Gaj et al., 2017). However, the drawback of their use in delivery is their tiny packing which make it essential to utilize multiple virions to deliver different components of CRISPER/Cas9 components (sgRNAs, Cas9 or donor DNA) which cause further decrease in efficiency (Yin et al., 2017). However, the utilization of AAV causes the incessant articulation of CRISPER components in editted cells which may cause 
unwanted off-target genome effects or may lead to increased immune response. Adenovirus and lentivirus can both infect dividing and non-dividing cells, but unlike lentiviruses, adenoviruses do not integrate into the genome of the recipient cell. Both lentiviruses and adenoviruses has their drawbacks as both induce strong immune response (Follenzi, Santambrogio, \& Annoni, 2007).

To solve these difficulties, non-viral delivery vectors or preassembled and short-lived Cas 9 RNP complexes can be used. Non-viral delivery approach uses liposomes, gold nanoparticle or inorganic nanoparticle and many others (Yin et al., 2014). Wide range of molecules are delivered for long through lipid nanoparticle and because they are devoid of any viral component so they lessen immunogenicity and security concerns and these nanoparticles can also be used both in vitro and in vivo. Additional benefits of using these nanoparticle for delivery of CRISPER/Cas9 components are their high loading capacity and the integration of genomic risk is not present and continuous articulation of CRISPER/Cas9 (Kaczmarek, Kowalski, \& Anderson, 2017). Researchers have documented successful provision of Cas9-sgRNA RNP compounds through nanoparticles in xenografts of U2OS human osteosarcoma cells (Sun et al., 2015). Moreover, Cas9 RNP complexes with donor DNA was shown to be delivered by gold nanoparticle combined with DNA a well as further added with disruptive polymers that are endosomal and cationic, could induce homology DNA repair (HDR) to fix DNA mutant variations of Duchenne muscular dystrophy in the mice(Lee et al., 2017). Goldnanoparticle is an excellent carrier that is not toxic for gene and drug delivery application because gold core of the particle provides solidity to the assemblage, meanwhile the monolayer permits surface tuning of properties like hydrophobicity and charge. There is still need for testing the safety and efficiency of this method, but it is expected CRISPER components delivery mechanism. As nanoparticles that are inorganic including bare mesoporus or dense silica nanoparticles and carbon nanotubes have already been used for various purposes, they are the natural and potential carriers of CRISPER components (Xu, Zeng, Lu, \& Yu, 2006).Furthermore, inorganic nanoparticles have some other benefits including their reproducible composition, size and stability over time as well as simplicity to generate them.

\section{CONCLUSION}

Although CRISPER/Cas9 based technology is still in development but it has already displayed its potential in research and hold great therapeutic promise but for favorable clinical application of this technology, secure and efficient transport into selected tissue is required. There are high expectations for this technology, which necessitated careful planning, such as allowing regulatory processes for its development. However, the technology still requires optimization mainly with respects to safety, specificity and efficacy before its widespread translation into clinics. Despite the many obstacles that must be met, we expect that the continued development of genetic science will significantly contribute to existing cancer therapies.

\section{REFERENCES}

[1] Adikusuma, F., Williams, N., Grutzner, F., Hughes, J., \& Thomas, P. (2017). Targeted Deletion of an Entire Chromosome Using CRISPR/Cas9. Molecular Therapy, 25(8), 1736-1738. https://doi.org/10.1016/j.ymthe.2017.05.021

[2] Aghi, M., Visted, T., DePinho, R. A., \& Chiocca, E. A. (2008). Oncolytic herpes virus with defective ICP6 specifically replicates in quiescent cells with homozygous genetic mutations in p16. Oncogene, 27(30), 4249-4254. https://doi.org/10.1038/onc.2008.53

[3] Ahmad, G., \& Amiji, M. (2018). Use of CRISPR/Cas9 gene-editing tools for developing models in drug discovery. Drug Discovery Today, 23(3), 519-533. https://doi.org/10.1016/j.drudis.2018.01.014

[4] Barrangou, R., Fremaux, C., Deveau, H., Richardss, M., Boyaval, P., Moineau, S., ... Horvath, P. (2007). CRISPR Provides Against Viruses in Prokaryotes. Science, 315(March), 1709-1712.

[5] Bolotin, A., Quinquis, B., Sorokin, A., \& Dusko Ehrlich, S. (2005). Clustered regularly interspaced short palindrome repeats (CRISPRs) have spacers of extrachromosomal origin. Microbiology, 151(8), 2551-2561. https://doi.org/10.1099/mic.0.28048-0

[6] Chavez, A., Tuttle, M., Pruitt, B. W., Ewen-Campen, B., Chari, R., Ter-Ovanesyan, D., ... Church, G. (2016). Comparison of Cas9 activators in multiple species. Nature Methods, 13(7), 563-567. https://doi.org/10.1038/nmeth.3871

[7] Chen, B., Gilbert, L. A., Cimini, B. A., Schnitzbauer, J., Zhang, W., Li, G. W., ... Huang, B. (2013). Dynamic imaging of genomic loci in living human cells by an optimized CRISPR/Cas system. Cell, 155(7), 1479-1491. https://doi.org/10.1016/j.cell.2013.12.001

[8] Choi, A. H., O'Leary, M. P., Fong, Y., \& Chen, N. G. (2016). From benchtop to bedside: A review of oncolytic virotherapy. Biomedicines, 4(3), 1-20. https://doi.org/10.3390/biomedicines4030018

[9] Choi, P. S., \& Meyerson, M. (2014). Targeted genomic rearrangements using CRISPR/Cas technology. Nature Communications, $5, \quad 1-6$. https://doi.org/10.1038/ncomms4728

[10] Cyranoski, D. (2016). CRISPR gene editing tested in a person. Nature, 539(7630), 479.

[11] Davis, K. M., Pattanayak, V., Thompson, D. B., Zuris, J. 
A., \& Liu, D. R. (2015). Small molecule-triggered Cas9 protein with improved genome-editing specificity. Nature Chemical Biology, 11(5), 316-318. https://doi.org/10.1038/nchembio.1793

[12] Daya, S., \& Berns, K. I. (2008). Gene therapy using adenoassociated virus vectors. Clinical Microbiology Reviews, 21(4), 583-593. https://doi.org/10.1128/CMR.00008-08

[13] Differ, G., Cobb, M. H., Fishman-lobell, J., \& Habert, J. E. (1992). Radl Nhej. 42580(September).

[14] Dominguez, A. A., Lim, W. A., \& Qi, L. S. (2016). Beyond editing: Repurposing CRISPR-Cas9 for precision genome regulation and interrogation. Nature Reviews Molecular Cell Biology, 17(1), 5-15. https://doi.org/10.1038/nrm.2015.2

[15] Dong, H., Strome, S. E., Salomao, D. R., Tamura, H., Hirano, F., Flies, D. B., ... Chen, L. (2002). Tumorassociated B7-H1 promotes T-cell apoptosis: A potential mechanism of immune evasion. Nature Medicine, 8(8), 793-800. https://doi.org/10.1038/nm730

[16] Fellmann, C., Gowen, B. G., Lin, P. C., Doudna, J. A., \& Corn, J. E. (2017). Cornerstones of CRISPR-Cas in drug discovery and therapy. Nature Reviews Drug Discovery, 16(2), 89-100. https://doi.org/10.1038/nrd.2016.238

[17] Fleuren, E. D. G., Zhang, L., Wu, J., \& Daly, R. J. (2016). The kinome "at large" in cancer. Nature Reviews Cancer, 16(2), 83-98. https://doi.org/10.1038/nrc.2015.18

[18] Follenzi, A., Santambrogio, L., \& Annoni, A. (2007). Immune Responses to Lentiviral Vectors. Current Gene Therapy, 7(5), 306-315. https://doi.org/10.2174/156652307782151515

[19] Friedland, A. E., Tzur, Y. B., Esvelt, K. M., Colaiácovo, M. P., Church, G. M., \& Calarco, J. A. (2013). Heritable genome editing in C. elegans via a CRISPR-Cas9 system. Nature $\quad$ Methods, 10(8), 741-743. https://doi.org/10.1038/nmeth.2532

[20] Gaj, T., Ojala, D. S., Ekman, F. K., Byrne, L. C., Limsirichai, P., \& Schaffer, D. V. (2017). In vivo genome editing improves motor function and extends survival in a mouse model of ALS. Science Advances, 3(12), 1-11. https://doi.org/10.1126/sciadv.aar3952

[21] Gasiunas, G., Barrangou, R., Horvath, P., \& Siksnys, V. (2012). Cas9-crRNA ribonucleoprotein complex mediates specific DNA cleavage for adaptive immunity in bacteria. Proceedings of the National Academy of Sciences of the United States of America, 109(39), 2579-2586. https://doi.org/10.1073/pnas.1208507109

[22] Gauthier, J., \& Turtle, C. J. (2018). Insights into cytokine release syndrome and neurotoxicity after CD19-specific CAR-T cell therapy. Current Research in Translational Medicine, 66(2), 50-52. https://doi.org/10.1016/j.retram.2018.03.003

[23] Gilbert, L. A., Horlbeck, M. A., Adamson, B., Villalta, J. E., Chen, Y., Whitehead, E. H., ... Weissman, J. S. (2014). Genome-Scale CRISPR-Mediated Control of Gene Repression and Activation. Cell, 159(3), 647-661. https://doi.org/10.1016/j.cell.2014.09.029

[24] Goldsmith, B. K., Chen, W., Johnson, D. C., \& Hendricks, R. L. (1998). Neurovirulence by Blocking the CD8+ T Cell
Response. The Journal of Experimental Medicine, 187(3), $0-7$.

[25] Guichard, S. M. (2017). CRISPR-Cas9 for Drug Discovery in Oncology. In Annual Reports in Medicinal Chemistry (1st ed., Vol. https://doi.org/10.1016/bs.armc.2017.08.006

[26] Guilinger, J. P., Thompson, D. B., \& Liu, D. R. (2014). Fusion of catalytically inactive Cas9 to FokI nuclease improves the specificity of genome modification. Nature Biotechnology, 32(6), 577-582. https://doi.org/10.1038/nbt.2909

[27] Ishino, Y., Shinagawa, H., Makino, K., Amemura, M., \& Nakatura, A. (1987). Nucleotide sequence of the iap gene, responsible for alkaline phosphatase isoenzyme conversion in Escherichia coli, and identification of the gene product. Journal of Bacteriology, 169(12), 5429-5433. https://doi.org/10.1128/jb.169.12.5429-5433.1987

[28] Iwai, Y., Ishida, M., Tanaka, Y., Okazaki, T., Honjo, T., \& Minato, N. (2002). Involvement of PD-L1 on tumor cells in the escape from host immune system and tumor immunotherapy by PD-L1 blockade. Proceedings of the National Academy of Sciences of the United States of America, 99(19), 12293-12297. https://doi.org/10.1073/pnas.192461099

[29] Jakočinas, T., Bonde, I., Herrgård, M., Harrison, S. J., Kristensen, M., Pedersen, L. E., ... Keasling, J. D. (2015). Multiplex metabolic pathway engineering using CRISPR/Cas9 in Saccharomyces cerevisiae. Metabolic Engineering, 28, 213-222. https://doi.org/10.1016/j.ymben.2015.01.008

[30] Jinek, M., Chylinski, K., Fonfara, I., Hauer, M., Doudna, J. A., \& Charpentier, E. (2012). A Programmable Dual-RNA - Guided. 337(August), 816-822.

[31] Joung, J., Konermann, S., Gootenberg, J. S., Abudayyeh, O. O., Platt, R. J., Brigham, M. D., ... Zhang, F. (2017). Genome-scale CRISPR-Cas9 knockout and transcriptional activation screening. Nature Protocols, 12(4), 828-863. https://doi.org/10.1038/nprot.2017.016

[32] Kaczmarek, J. C., Kowalski, P. S., \& Anderson, D. G. (2017). Advances in the delivery of RNA therapeutics: From concept to clinical reality. Genome Medicine, 9(1), 1-16. https://doi.org/10.1186/s13073-017-0450-0

[33] Kansagra, A., \& Litzow, M. (2017). Treatment of Young Adults with Acute Lymphoblastic Leukemia. Current Hematologic Malignancy Reports, 12(3), 187-196. https://doi.org/10.1007/s11899-017-0377-y

[34] Kay, M. A. (2011). State-of-the-art gene-based therapies: The road ahead. Nature Reviews Genetics, 12(5), 316-328. https://doi.org/10.1038/nrg2971

[35] Kim, S., Kim, D., Cho, S. W., Kim, J., \& Kim, J. S. (2014). Highly efficient RNA-guided genome editing in human cells via delivery of purified Cas 9 ribonucleoproteins. Genome Research, 24(6), 1012-1019. https://doi.org/10.1101/gr.171322.113

[36] Klann, T. S., Black, J. B., Chellappan, M., Safi, A., Song, L., Hilton, I. B., ... Gersbach, C. A. (2017). CRISPR-Cas9 epigenome editing enables high-throughput screening for functional regulatory elements in the human genome. 
Nature Biotechnology, 35(6), 561-568. https://doi.org/10.1038/nbt.3853

[37] Kleinstiver, B. P., Pattanayak, V., Prew, M. S., Tsai, S. Q., Nguyen, N. T., Zheng, Z., \& Joung, J. K. (2016). Highfidelity CRISPR-Cas9 nucleases with no detectable genome-wide off-target effects. Nature, 529(7587), 490495. https://doi.org/10.1038/nature16526

[38] Konermann, S., Brigham, M. D., Trevino, A. E., Joung, J., Abudayyeh, O. O., Barcena, C., ... Zhang, F. (2015). Genome-scale transcriptional activation by an engineered CRISPR-Cas9 complex. Nature, 517(7536), 583-588. https://doi.org/10.1038/nature14136

[39] Kotterman, M. A., \& Schaffer, D. V. (2014). Engineering adeno-associated viruses for clinical gene therapy. Nature Reviews Genetics, 15(7), 445-451. https://doi.org/10.1038/nrg3742

[40] Lee, K., Conboy, M., Park, H. M., Jiang, F., Kim, H. J., Dewitt, M. A., ... Murthy, N. (2017). Nanoparticle delivery of Cas9 ribonucleoprotein and donor DNA in vivo induces homology-directed DNA repair. Nature Biomedical Engineering, $1(11)$ 889-901. https://doi.org/10.1038/s41551-017-0137-2

[41] Li, W., Teng, F., Li, T., \& Zhou, Q. (2013). Simultaneous generation and germline transmission of multiple gene mutations in rat using CRISPR-Cas systems. Nature Biotechnology, 31(8), 684-686. https://doi.org/10.1038/nbt.2652

[42] Liang, F., Han, M., Romanienko, P. J., \& Jasin, M. (1998). Homology-directed repair is a major double-strand break repair pathway in mammalian cells. Proceedings of the National Academy of Sciences of the United States of America, 95(9), 5172-5177. https://doi.org/10.1073/pnas.95.9.5172

[43] Luo, J. (2016). CRISPR/Cas9: From Genome Engineering to Cancer Drug Discovery. Trends in Cancer, 2(6), 313324. https://doi.org/10.1016/j.trecan.2016.05.001

[44] Maeder, M. L., Linder, S. J., Cascio, V. M., Fu, Y., Ho, Q. H., \& Joung, J. K. (2013). CRISPR RNA-guided activation of endogenous human genes. Nature Methods, 10(10), 977-979. https://doi.org/10.1038/nmeth.2598

[45] Makarova, K. S., Haft, D. H., Barrangou, R., Brouns, S. J. J., Charpentier, E., Horvath, P., ... Koonin, E. V. (2011). Evolution and classification of the CRISPR-Cas systems. Nature Reviews Microbiology, 9(6), 467-477. https://doi.org/10.1038/nrmicro2577

[46] Mali, P., Esvelt, K. M., \& Church, G. M. (2013). Cas9 as a versatile tool for engineering biology. Nature Methods, 10(10), 957-963. https://doi.org/10.1038/nmeth.2649

[47] Maus, M. V, Grupp, S. A., Porter, D. L., \& June, C. H. (2014). ANTIBODY DERIVATIVES AS NEW THERAPEUTICS FOR HEMATOLOGIC Antibody-modi fi ed $T$ cells: CARs take the front seat for hematologic malignancies. 123(17), 2625-2635. https://doi.org/10.1182/blood-2013-11-492231.organs

[48] Mojica, F. J. M., Díez-Villaseñor, C., García-Martínez, J., \& Almendros, C. (2009). Short motif sequences determine the targets of the prokaryotic CRISPR defence system. Microbiology, 155(3), 733-740. https://doi.org/10.1099/mic.0.023960-0

[49] Nihongaki, Y., Kawano, F., Nakajima, T., \& Sato, M. (2015). Photoactivatable CRISPR-Cas9 for optogenetic genome editing. Nature Biotechnology, 33(7), 755-760. https://doi.org/10.1038/nbt.3245

[50] Oakes, B. L., Nadler, D. C., Flamholz, A., Fellmann, C., Staahl, B. T., Doudna, J. A., \& Savage, D. F. (2016). Profiling of engineering hotspots identifies an allosteric CRISPR-Cas9 switch. Nature Biotechnology, 34(6), 646651. https://doi.org/10.1038/nbt.3528

[51] Ran, F. A., Hsu, P. D., Lin, C. Y., Gootenberg, J. S., Konermann, S., Trevino, A. E., ... Zhang, F. (2013). XDouble nicking by RNA-guided CRISPR cas9 for enhanced genome editing specificity. Cell, 154(6), 1-10. https://doi.org/10.1016/j.cell.2013.08.021

[52] Ribeiro, N. (2014)

\section{主観的健康感を中心とした在宅高齢者における} 健康関連指標に関する共分散構造分析Title.

343(January), 84-88.

[53] Scott, A. (2018). A CRISPR path to drug discovery. Nature, 555(7695), $\quad$ S10-S11. https://doi.org/10.1038/d41586-018-02477-1

[54] Shachaf, C. M., Kopelman, A. M., Arvanitis, C., Karlsson, A., Beer, S., Mandl, S., ... Felsher, D. W. (2004). MYC inactivation uncovers pluripotent differentiation and tumour dormancy in hepatocellular cancer. Nature, 431(7012),

1112-1117. https://doi.org/10.1038/nature03043

[55] Slaymaker, I. M., Gao, L., Zetsche, B., Scott, D. A., Yan, W. X., \& Zhang, F. (2016). Rationally engineered Cas9 nucleases with improved specificity. Science, 351(6268), 84-88. https://doi.org/10.1126/science.aad5227

[56] Smurnyy, Y., Cai, M., Wu, H., McWhinnie, E., Tallarico, J. A., Yang, Y., \& Feng, Y. (2014). DNA sequencing and CRISPR-Cas9 gene editing for target validation in mammalian cells. Nature Chemical Biology, 10(8), 623625. https://doi.org/10.1038/nchembio.1550

[57] Su, S., Hu, B., Shao, J., Shen, B., Du, J., Du, Y., ... Liu, B. (2016). CRISPR-Cas9 mediated efficient PD-1 disruption on human primary $\mathrm{T}$ cells from cancer patients. Scientific Reports, 6, 1-13. https://doi.org/10.1038/srep20070

[58] Sun, W., Ji, W., Hall, J. M., Hu, Q., Wang, C., Beisel, C. L., \& Gu, Z. (2015). Self-Assembled DNA Nanoclews for the Efficient Delivery of CRISPR-Cas9 for Genome Editing. Angewandte Chemie - International Edition, 54(41), 12029-12033. https://doi.org/10.1002/anie.201506030

[59] Torres-Ruiz, R., Martinez-Lage, M., Martin, M. C., Garcia, A., Bueno, C., Castaño, J., ... Rodriguez-Perales, S. (2017). Efficient Recreation of $\mathrm{t}(11 ; 22)$ EWSR1-FLI1+ in Human Stem Cells Using CRISPR/Cas9. Stem Cell Reports, 8(5), 1408-1420. https://doi.org/10.1016/j.stemcr.2017.04.014

[60] Torres, R., Garcia, A., Jimenez, M., Rodriguez, S., \& Ramirez, J. C. (2014). An integration-defective lentivirusbased resource for site-specific targeting of an edited safeharbour locus in the human genome. Gene Therapy, 21(4), 343-352. https://doi.org/10.1038/gt.2014.1

[61] Torres, R., Martin, M. C., Garcia, A., Cigudosa, J. C., 
Ramirez, J. C., \& Rodriguez-Perales, S. (2014). Engineering human tumour-associated chromosomal translocations with the RNA-guided CRISPR-Cas9 system. Nature Communications, 5, 1-8. https://doi.org/10.1038/ncomms4964

[62] Tsai, S. Q., \& Joung, J. K. (2016). Defining and improving the genome-wide specificities of CRISPR-Cas9 nucleases. Nature Reviews Genetics, 17(5), 300-312. https://doi.org/10.1038/nrg.2016.28

[63] Tseng, S. Y., Otsuji, M., Gorski, K., Huang, X., Slansky, J. E., Pai, S. I., ... Tsuchiya, H. (2001). B7-DC, a new dendritic cell molecule with potent costimulatory properties for T cells. Journal of Experimental Medicine, 193(7), 839-845. https://doi.org/10.1084/jem.193.7.839

[64] Tsushima, F., Yao, S., Shin, T., Flies, A., Flies, S., Xu, H., ... Chen, L. (2007). Interaction between B7-H1 and PD-1 determines initiation and reversal of T-cell anergy. Blood, 110(1), 180-185. https://doi.org/10.1182/blood-2006-11060087

[65] van der Oost, J., Jore, M. M., Westra, E. R., Lundgren, M., \& Brouns, S. J. J. (2009). CRISPR-based adaptive and heritable immunity in prokaryotes. Trends in Biochemical Sciences, 34(8), 401-407. https://doi.org/10.1016/j.tibs.2009.05.002

[66] Van Soolingen, D., De Haas, P. E. W., Hermans, P. W. M., Groenen, P. M. A., \& Van Embden, J. D. A. (1993). Comparison of various repetitive DNA elements as genetic markers for strain differentiation and epidemiology of Mycobacterium tuberculosis. Journal of Clinical Microbiology, 31(8), 1987-1995. https://doi.org/10.1128/jcm.31.8.1987-1995.1993

[67] Walton, J., Blagih, J., Ennis, D., Leung, E., Dowson, S., Tookman, L. A., ... Vousden, K. (2016). CRISPR/Cas9mediated.

[68] Wang, T., Wei, J. J., Sabatini, D. M., \& Lander, E. S. (2014). Genetic screens in human cells using the CRISPRCas9 system. Science, 343(6166), 80-84. https://doi.org/10.1126/science.1246981

[69] Wyman, J., Changeux, J. P., Filmer, D., Jovin, T. M., Baehr, W., Holbrook, J. J., ... Bustamante, C. (2013). Multiplex Genome Engineering Using CRISPER/Cas System. Science, (February), 819-824.

[70] Xu, Z. P., Zeng, Q. H., Lu, G. Q., \& Yu, A. B. (2006). Inorganic nanoparticles as carriers for efficient cellular delivery. Chemical Engineering Science, 61(3), 10271040. https://doi.org/10.1016/j.ces.2005.06.019

[71] Yin, H., Kanasty, R. L., Eltoukhy, A. A., Vegas, A. J., Dorkin, J. R., \& Anderson, D. G. (2014). Non-viral vectors for gene-based therapy. Nature Reviews Genetics, 15(8), 541-555. https://doi.org/10.1038/nrg3763

[72] Yin, H., Kauffman, K. J., \& Anderson, D. G. (2017). Delivery technologies for genome editing. Nature Reviews Drug Discovery, 16(6), 387-399. https://doi.org/10.1038/nrd.2016.280

[73] Yin, H., Song, C. Q., Dorkin, J. R., Zhu, L. J., Li, Y., Wu, Q., ... Anderson, D. G. (2016). Therapeutic genome editing by combined viral and non-viral delivery of CRISPR system components in vivo. Nature Biotechnology, 34(3),
328-333. https://doi.org/10.1038/nbt.3471 